\title{
Neurogenesis in the Dentate Gyrus of the Hippocampus Associated with Sex Hormone Levels in Female Mice during Different Stages of the Estrous Cycle
}

\section{Dana $\mathrm{P}^{* 1}$ and Ghorbanian $\mathrm{MT}^{2}$}

${ }^{1}$ M.Sc, Histology and Embryology, Damghan University School of Science, Damghan, Iran

${ }^{2}$ Assistant prof, in anatomical science, Damghan University School of Science, Damghan, Iran

${ }^{*}$ Corresponding author: Dana P, M.Sc, Histology and Embryology, Damghan University School of Science, Damghan, Iran, Tel: 09120195880, E-mail: parisadana95@gmail.com

Citation: Dana P, Ghorbanian MT (2018) Neurogenesis in the Dentate Gyrus of the Hippocampus Associated with Sex Hormone Levels in Female Mice During Different Stages of the Estrous Cycle. J Cell Biol Histol 1(1): 105. doi: 10.15744/2638-082X.1.105

Received Date: March 20, 2018 Accepted Date: June 06, 2018 Published Date: June 08, 2018

\begin{abstract}
Background: Usual fluctuations of sex hormones during the estrous cycle in female mice affect the hippocampal neurogenesis. The hippocampus also comprises many steroid receptors suggesting the modulatory effects of gonadal hormones on the hippocampal plasticity. Sex differences at the level of structure and function of the hippocampus are mostly due to the fluctuations of steroid hormones during the estrous cycle. The purpose of this study was to investigate relationship with serum sex hormone levels and the expression of glial fibrillary acidic protein (GFAP) at different phases of the estrous cycle.
\end{abstract}

Methods: In this study, different stages of the estrous cycle were determined in age 6-8 week NMRI female mice by methylene bluestained vaginal smears, Were investigated astrocytes in the hippocampus using GFAP immunohistochemistry and Sex hormone levels were Measured by ELISA kit (measuring hormonal levels a control).

Results: GFAP immunostaining showed the changes in the morphology of astrocytes. The GFAP-positive granular sub-region focused on Proestrus stage. GFAP positive in estrous phase is concentrated around the vessels; prolactin concentration is Maximum at this stage. Metestrus and Diestrus stage Hilus GFAP in the region is concentrated.

Conclusion: In this study Hippocampal Immunohistochemistry for GFAP markers were evaluated. The highest number of cells and staining in the Proestrus stage and were among the lowest in Estrous and Diestrus, Metestrus.

Keywords: Neurogenesis; Hippocampus; Estrous Cycle; Estrogen; Progesterone

\section{Introduction}

Each year, psychiatric disorders such as depression and anxiety affect millions of people worldwide. Researchers believe that the risk of mental disorders among women is almost twice that of men, some of the emotional and mood disorders linked by menstrual cycle of women that is moderated by ovarian steroids [1,2]. Neurogenesis and neuronal cells in the brain is influenced by steroids. Steroids effect on the expression of glial cell including astrocytes. Identification marker GFAP astrocytes and hippocampal stem cells. Studies have shown that increased Estrogen on the neural stem cells in dentate gyrus of the hippocampus with changes in hormone dose is changed and the other is progesterone hormone effect on cell proliferation in the region. Tanapat Research and his colleagues in 2005 on ovarectomy rats that are subjected to high levels of estrogen Proliferation of stem cells in the hippocampus showed, While at the same time being exposed to progesterone and estrogen inhibits cell proliferation induced by estrogen, Set in front of neurogenesis induced by progesterone alone will increase cell proliferation in vitro $[3,4]$. Estrous cycle is repeated periodically mammalian sexual season. Estrus is female libido. The name comes from the Greek word meaning insanity or madness has been oistros to that described Describing sexual material [5].

Estrous cycle has four consecutive stages, including 1.Proestrus 2.Estrus 3.Metestrus 4.Diestrus. Is a Proestrus (follicular development, endometrial proliferation) Step by increasing body temperature is female. Estrus meant preparation animal adoption male and female for mating. Metestrus (differentiated endometrial) is a short period of activity of reproductive organs gradually 
subside during the period. The Terminal stage is the Diestrus. Anestrus, non-reproductive season is a period of rest. Estrous cycle in mice occurs every 4 to 5 days with increasing concentrations of estrogen and progesterone [6,7]. In the dentate gyrus female rats, a cell proliferation fluctuation during the estrous cycle has been observed. The number of new cells in the Proestrus stage and the highest decreases in ovarectomy, Although estrogen replacement number of new cells compared to intact controls return to normal operation [8]. By contrast estrous cycle ovarian hormones and pituitary hormones (FSH and LH) are controlled. Peptide hormones derived from the pituitary hormone, follicle-stimulating hormone and luteal ovarian hormones with steroid hormones, estrogen and progesterone are the main controller estrous cycle. Gennady natural fluctuation of hormones during the Estrous cycle in mice affects the hippocampus neurogenesis. Four to five days during the Estrous cycle in rodents, in Proestrus stage, constantly increases circulating estrogen. The next day estrous cycle stage and the stage of Diestrus slightly lower circulating estrogen raises for only return to Proestrus, the speed increases $[9,10]$. Prolactin hormone is secreted from the anterior pituitary and hypothalamic factors such as TRH to stimulate the production of prolactin. Previous studies was that the researchers of PRL receptor in the choroid plexus and the posterior region of the adult SVZ and in the front part of the brain can be expressed, but new research using antibody BrdU showed that PRL and LH receptors in dentate gyrus and ovarian tissue has receptors are expressed. Two hormones LH and PRL are mediated by an increase in neurogenesis, when the concentration of high LH in Proestrus SVZ and SGZ receptor is expressed in both the neurogenic region [11]. In the basophil cells anterior pituitary LH hormone is made and $\mathrm{GnRH}$ hormone secreted by the hypothalamus is controlled. These hormones create integration between white matter connections between the frontal and frontal cortical regions involved. In addition, the flexibility of astrocytes in the hypothalamus is under the influence of LH wave [12]. LH hormones directly affect brain morphology. Many physiological and behavioral effects of ovarian hormones estrogen and progesterone mediated apply. Fluctuations in estrogen levels during the Estrous cycle and increased $\mathrm{LH}$ concentrations of estrogen and progesterone is associated with a regulatory role. The specific roles of progesterone in the regulation of hippocampal neurons are not entirely clear, but signs of hippocampal neurogenesis by these hormones estradiol on their performance [13].

\section{Materials and methods}

\section{Reagents}

All materials from Aldrich - SIG-MA (Hamburg, Germany), respectively, except as separately listed.

\section{Animals}

The study included 56 female mice (6-8 week) used belonged to Naval Medical Research Institute (NMRI), housed and bred in accordance with the Guide for Care and Use of Laboratory Animals of Damghan University Pets under 12 hours of light and 12 hours of darkness and the temperature and humidity controlled (temperature $24-20^{\circ} \mathrm{C}$, humidity $60-50$ percent) were stored. They were given food and water enough. The approximate weight of the female rats was measured between 16-23 grams; Mice have been selected to be the same weight and same age the error rate and fluctuations in sex hormones during the estrous cycle in mice to a minimum. (Work on animal ethics on the agenda of the Ethics Committee, Faculty of Biology, University of Damghan was taken into consideration).

\section{Determination groups}

The action pipetting the vagina with saline solution three to five times slowly does the final solution vaginal discharge on a glass slide're dispreaded. After preparing the smear slides, dried at room temperature and we fixed for 5 minutes with methanol, the slides are stained with methylene blue two percent and with optical microscopy we observed. The epithelial cells were observed in estrous cycle phases to be determined. The mice in two separate categories, each category consists of four groups $(\mathrm{n}=7)$ Proestrus, Estrous, Metestrus and Diestrus is put.

\section{Morphological}

Animal by intraperitoneal injection Mix a solution of $100 \mathrm{mg}$ and $10 \mathrm{mg}$ ketamine xylazine per $\mathrm{kg}$ of body weight were anesthetized deep and after fixing mice and open the chest, needle through the heart into the aorta was, we cut the right atrium to drain blood with scissors. Cardiac perfusion with phosphate buffer solution, followed by $4 \%$ paraformaldehyde solution cool, Perfusion was performed and the brain was removed from the skull. After fixation and tissue processing steps and colonization, tissues in liquid paraffin $60{ }^{\circ} \mathrm{C}$ Cast and were transferred to the fridge for cool down and using a rotary microtome (Microtec model cut4055) coronal slices 5 micro were prepared, Tissues at the level of bregma (nearly $3 / 7 \mathrm{~mm}$ Plate anterior hippocampus including grainy areas gyrus) cut and to remove wrinkles; strips of tissue taken from $45^{\circ} \mathrm{C}$ water bath and smeared on slides glassy were covered with albumin. Samples were dried at room temperature and pre-painted slides, for an hour in the oven (Memmert, Germany) 50 ${ }^{\circ} \mathrm{C}$ were to be melted paraffin (Figure 1).

\section{Immunohistochemistry study}

Sequential $15 \mu \mathrm{m}$ coronal sections were cut and mounted on Silane (g-methacryloxypropyltri-methoxysilane, SIGMA, St. Louis, MO, USA) covered slides and were processed for double immunofluorescence staining. Briefly, slides containing $15 \mu \mathrm{m}$ sections 


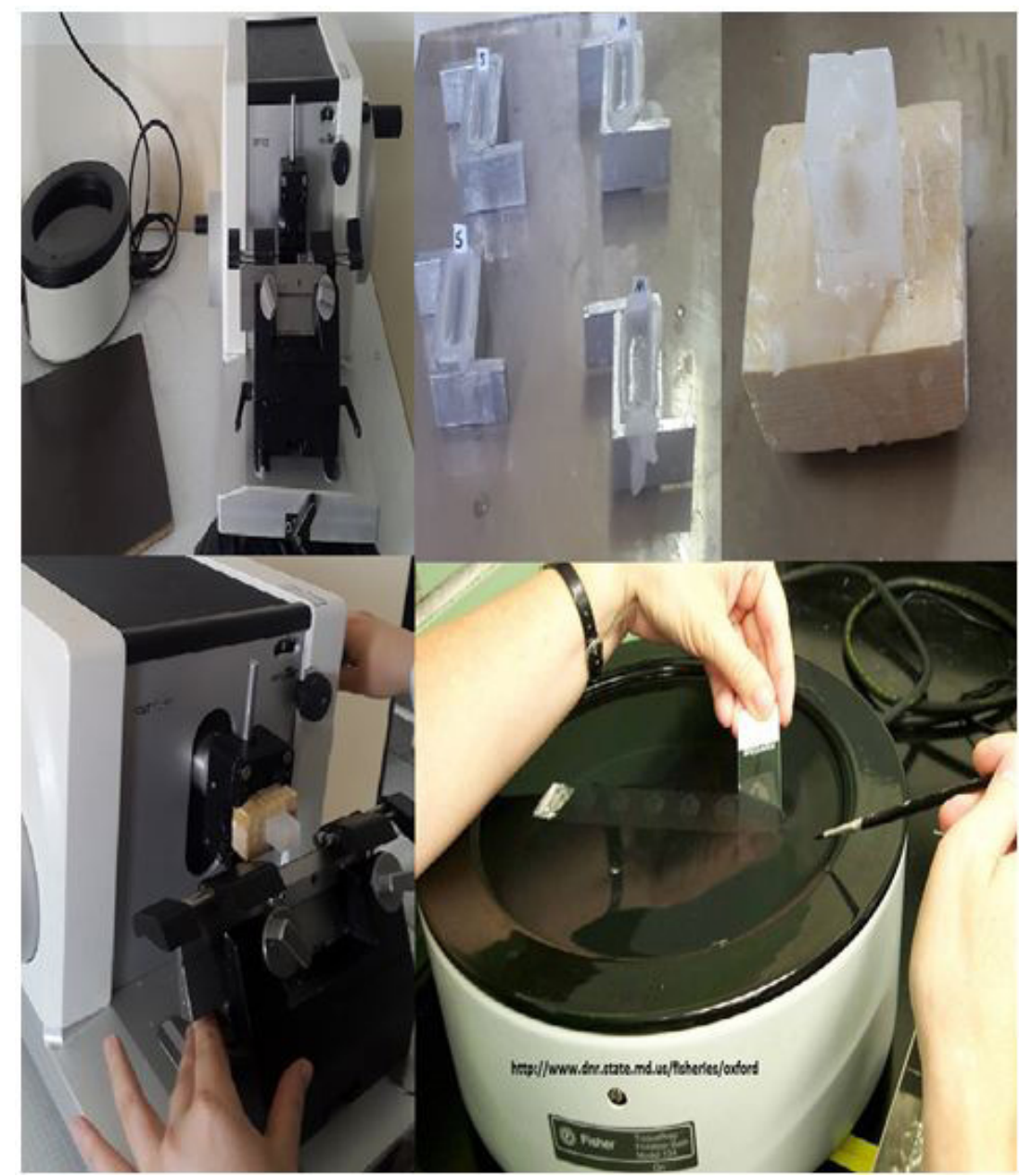

Figure 1: Molding and cutting of the mouse brain .molding and was transferred to the fridge to cool and then use the rotary microtome 5 micron coronal slices were prepared

were placed in a vacuum chamber for $20 \mathrm{~min}$ before and after mounting the sections. Once the slices were dry, sections were washed with PBS-Triton $(0.3 \%)$ three times, 10 min each $(3 \times 10)$, and were incubated with normal horse serum (NHS)(1:25) (Vector, Burlingame, CA, USA) in PBS-Triton (0.3\%) for $2 \mathrm{~h}$. Afterwards, they were incubated in anti-Glial Fibrillary Acidic Protein (GFAP) (mouse IgG1 isotype) (1:200; Sigma-Aldrich) for $24 \mathrm{~h}$ in a humid chamber at $4{ }^{\circ} \mathrm{C}$. Sections were washed $3 \times 10$ with PBS-Triton (0.3\%). and Goat Anti-Mouse IgG affinity purified, Rhodamine conjugate (1:200; Cat No: AP 124R) for 3 min in humid chamber. Afterwards, they were washed $3 \times 10$ in PBS and covered with fluorescent mounting medium (DAKO, Carpinteria, CA, USA). Negative controls consisted of eliminating the primary antibody for the procedure. To insure identical immunolabeling between groups, slides containing sections from each group were processed in parallel.

We see slides with fluorescent microscope. Microscopic images with a fluorescent microscope Japan, Nikon Eclipse, E600, Japan and digital camera imaging system using DXM 120, USA Nikon was done. The density of GFAP-positive cells in the hippocampus dentate gyrus was evaluated with the software ImageJ.

\section{Blood samples from mice}

Since a large amount of blood required to be removed after the animal sampling method used cardiac puncture. Mice under deep anesthesia with a needle No. 24 on the left side of the chest between the ribs five and six passed, and driven to the heart and As can be easily opened chest surgery is done to collect blood from the heart. Blood samples in a centrifuge refrigerated at $4{ }^{\circ} \mathrm{C}$ for 15 minutes to give away at 8 thousand. Blood serum was then on we separated with the help of micro pipette.

\section{Hormonal assay}

ELISA method was used to measure sexual hormones. Base Kit on the method Sandwich and is using monoclonal antibodies, using a spectrophotometer absorbance at $450 \mathrm{~nm}$ measures. Kit estrogen measurement of Dia Metra and progesterone kit was purchased from IBL International GmbH, assays kit prolactin, FSH, LH from company Pishtaz Teb purchased.

\section{Statistical method}

For studying morphological differences, we conducted measurements of astrocytic area calculated by using Image Pro Plus. 
Gray scale images exclusively containing astrocytes were obtained after separating the channel corresponding to the 550-570 $\mathrm{nm}$ wavelength excitation filter form the original image. Automatic object recognition was applied and the number of astrocytes as well as the area of each detected astrocyte was automatically calculated. Special care was taken to include in the analysis only complete astrocytes and not fragments. Immunoreactivity data were analyzed by number of sections in each group, and those of astrocytic area were analyzed by number of cells. All data were analyzed by using a one-way analysis of variance (ANOVA) followed by a post hoc analysis with a Sheffe's test. All comparisons were performed using SPSS 12.0 software for Windows. Values of p b 0.05 were considered statistically significant.

\section{Results}

Vaginal smear: (A) Proestrus stage: nucleated epithelial cells are found in abundance. (B) Estrous stage: horny epithelial cells with no nucleus are plentiful. (C) Stage of Metestrus: epithelial cells without nuclei and abundant neutrophils can be seen. (D) Stage of Diestrus: neutrophils, the most abundant found (Figure 2).
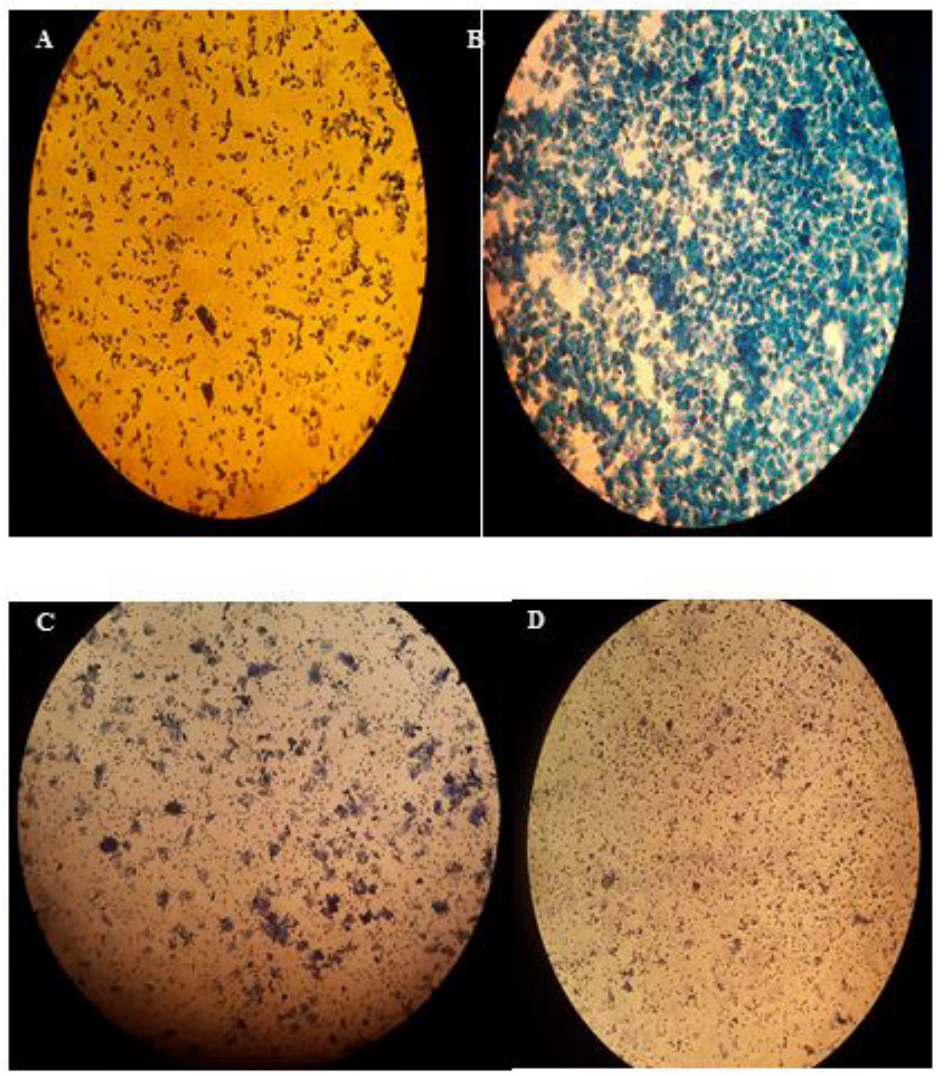

Figure 2: The Estrous cycle phases Vaginal smear. (A) The stage of Proestrus: nucleated epithelial cells are found in abundance, (B) phase-Estrus: horny epithelial cells with no nucleus are plentiful , (C) stage of Metestrus: epithelial cells without nuclei and abundant neutrophils can be seen (D) stage of Diestrus: neutrophils, the most abundant found

\section{The results of histological observations}

The stage Proestrus marker GFAP expression in the area Hilus dentate gyrus cell body can be seen with multiple processes is concentrated in the SGZ. Split along the stained cells in between Gyrus granular cell area is clearly visible, in the area of molecular GFAP staining positive cells as well as the morphology of the cells and their excrements show, in the area of perivascular astrocytes completely engulfed vessels and for such rings can be seen. SGZ staining intensity in Proestrus Compared to the other groups were higher, in the area of molecular marker GFAP response was markedly reduced (Figure 3 ).

The stage Estrous marker GFAP expression in cell morphology clearly different Proestrus stage, Focus GFAP-positive cells in the central region Hilus more than SGZ area, GFAP staining not like the Proestrus stage and the less visible grain gyrus cells, in the area of molecular cell marker GFAP more reactive towards perivascular astrocytes more positive GFAP-cell areas around the vessels (Figure 4).

The stage Matestrus the distribution of GFAP-positive cells in the dentate gyrus is visible throughout the region Hilus. Cells that were clearly seen in the SGZ the region and excrements of these cells in the gyrus granular cell extends to the area of molecular arrives, Less excrements branched cells and cell processes stretches without branching, In the area of molecular and GFAP positive cells are seen with frills long and sometimes bifurcated. The morphology of cells staining with their tributaries will showcase typical astrocytes (Figure 5). 


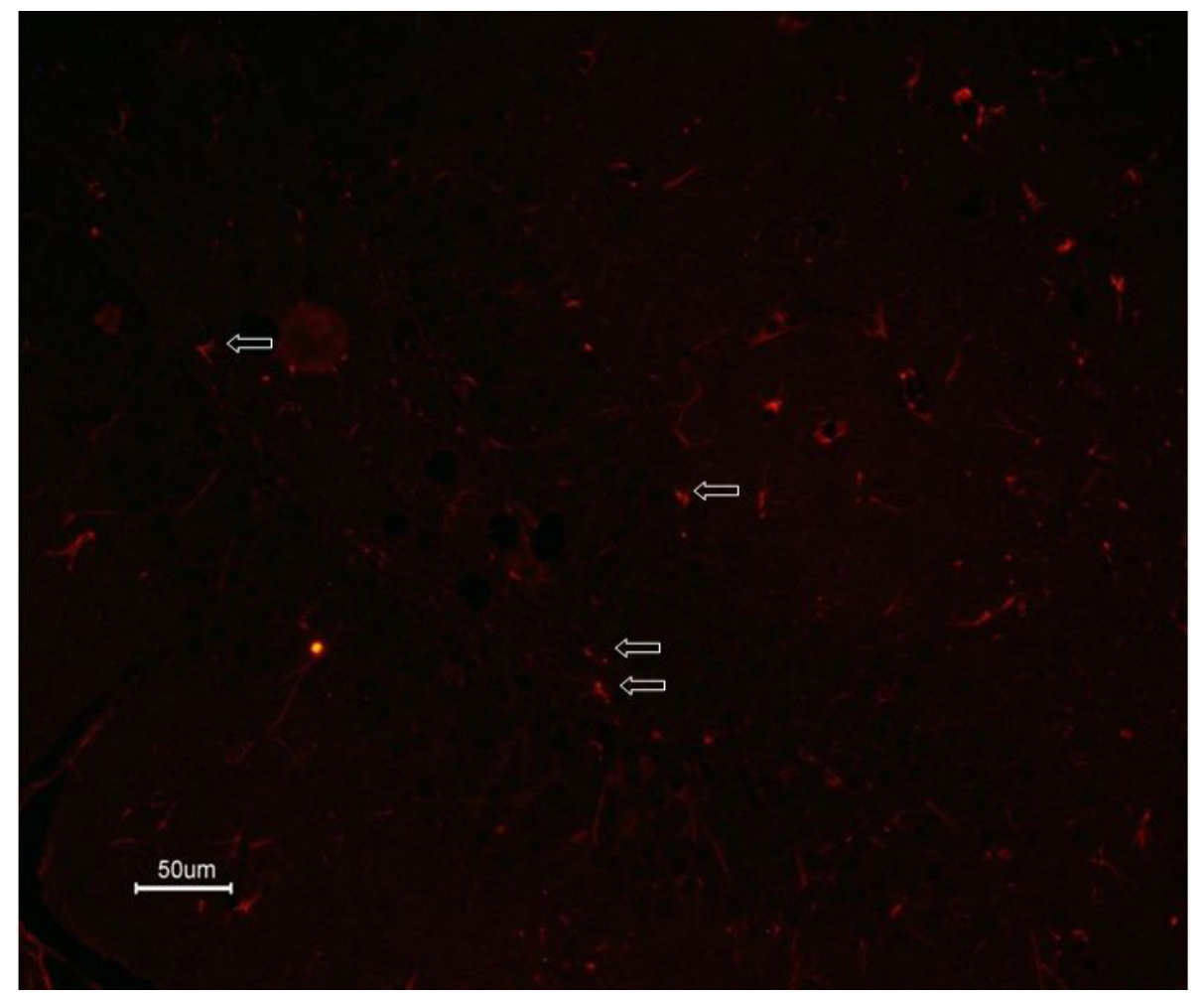

Figure 3: The thin sections of mouse hippocampus using GFAP immunohistochemistry markers. Thin sections of rat hippocampus in Proestrus stage, Arrow GFAP positive SGZ show

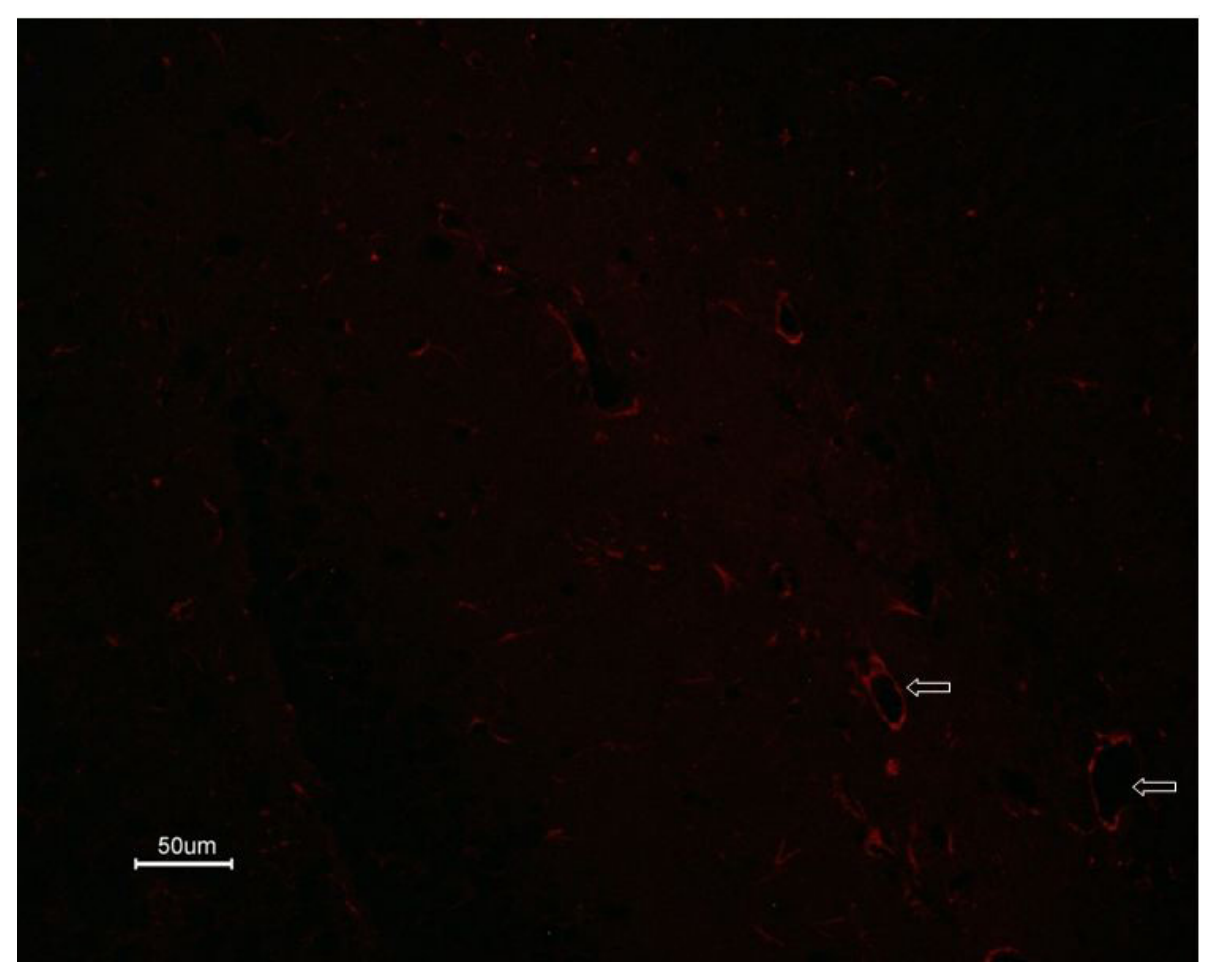

Figure 4: The thin sections of mouse hippocampus using GFAP immunohistochemistry markers. Thin sections of rat hippocampus in Estrous stage, perivascular astrocytes Arrow show

The stage Diestrus Marker GFAP expression in the region Hilus for the bright red color can be seen, these spots are scattered throughout Hilus and only a few cell morphology with their Excrement is visible. GFAP-positive cells in the SGZ cannot be detected. The Proestrus cells with characteristic morphology seen in SGZ and their processes to the granular layer extends gyrus, In Estrous and Diestrus these processes is less visible and different in the area of molecular cell dentate gyrus GFAP positive astrocytes are representative, staining good show and their morphology is quite evident and star-shaped cells with five or six 
separate branch from the cell body can be seen (Figure 6 and 7).

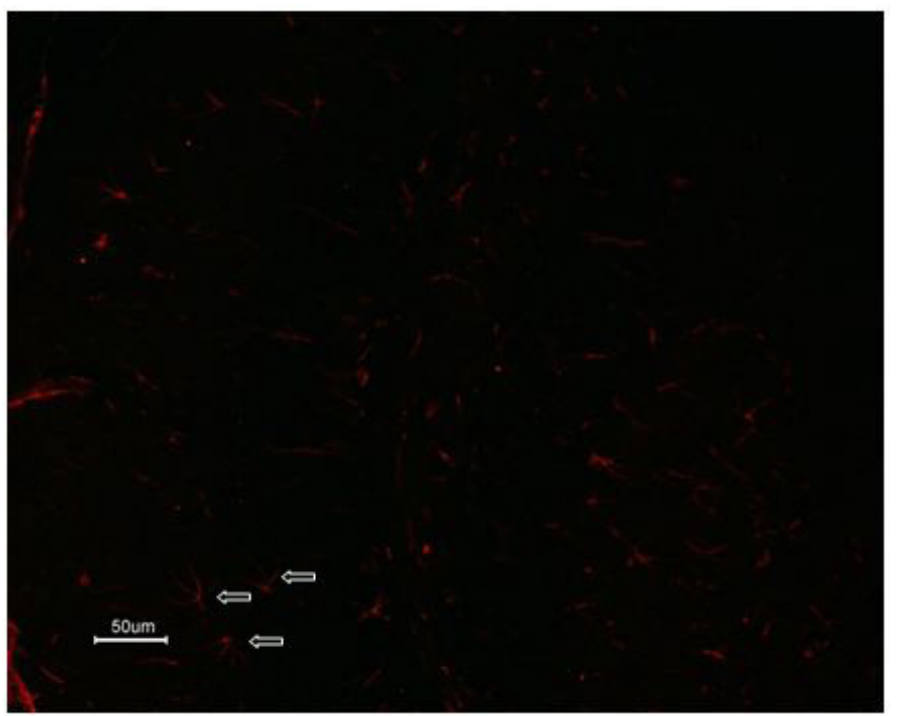

Figure 5: The thin sections of mouse hippocampus using GFAP immunohistochemistry markers. Thin sections of mouse hippocampus stage of Metestrus, star-shaped astrocytes arrows indicate the molecular layer

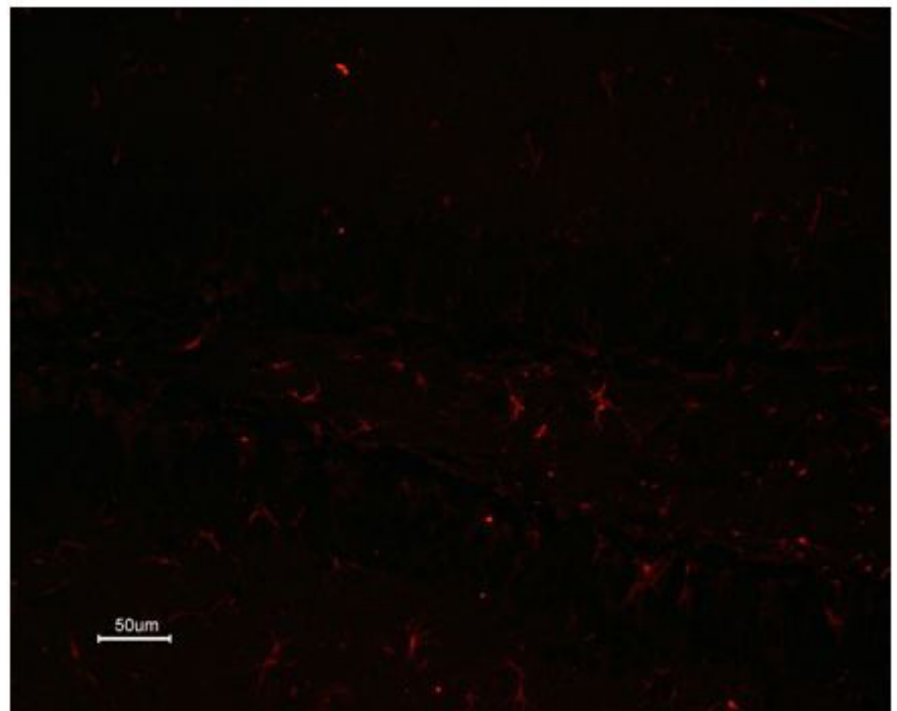

Figure 6: The thin sections of mouse hippocampus using GFAP immunohistochemistry markers .thin sections of rat hippocampus in Diestrus stage, star-shaped arrows show Hilus and molecular layer

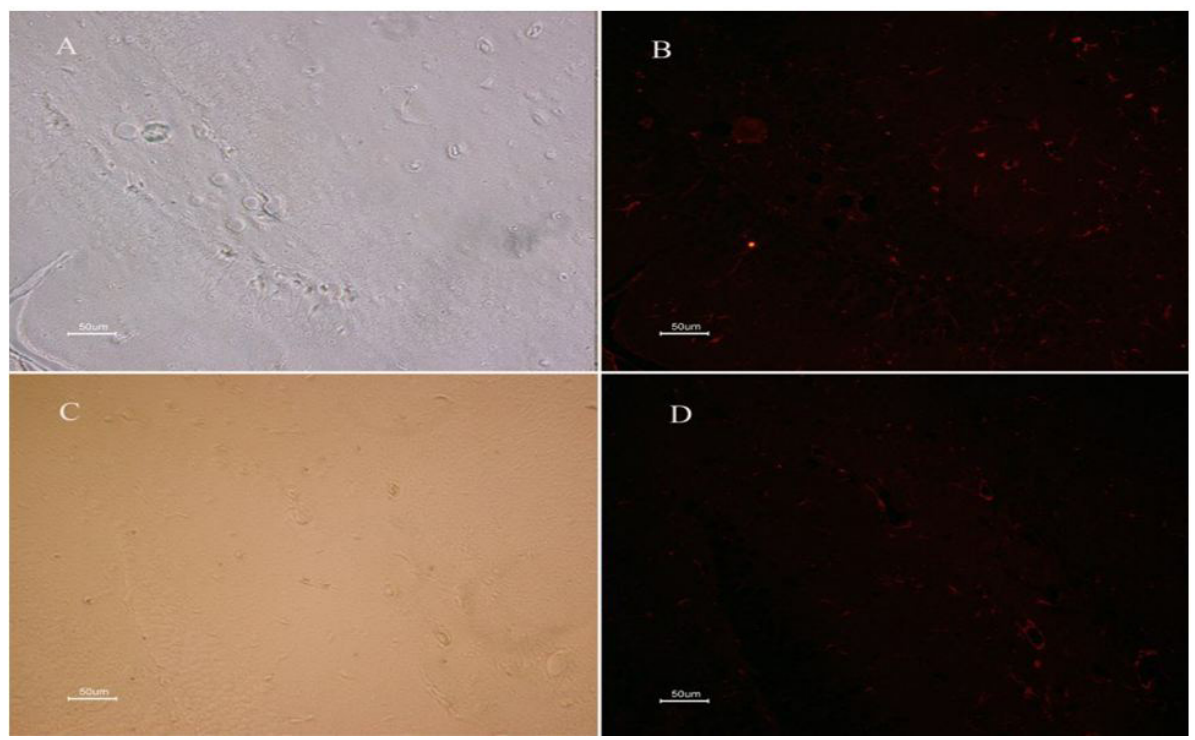



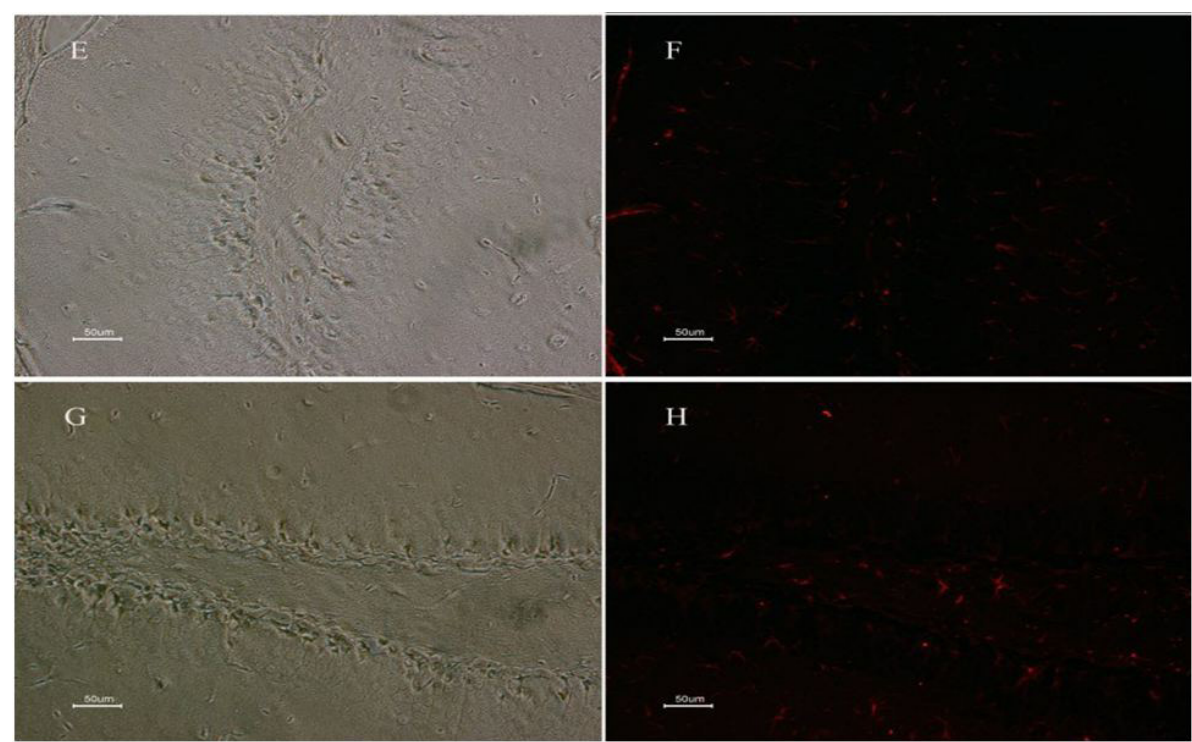

Figure 7: The microscopic sections of the hippocampus of mice at different stages of the estrous cycle, Proestrus (A, B), Estrus $(\mathrm{C}, \mathrm{D})$, Metestrus (E, F), Diestrus $(\mathrm{G}, \mathrm{H})$, phase contrast image on the left is the right immunofluorescent images

\section{Statistical comparison of the density of GFAP marker in the stages of estrous cycle}

In terms of density marker GFAP two Proestrus and Estrus stage with no significant differences ( $\mathrm{p}<0.05$ ), but with two stages Metestrus and Diestrus are significant. The highest number of cell marker GFAP staining in Proestrus stage and the lowest density in the area SGZ Metestrus and Diestrus was on stage, Most of cell proliferations in mice Occur Proestrus stage, which is 50\% above the stage of Diestrus. GFAP-positive cells in the hippocampus dentate gyrus SGZ Proestrus stage with high redundancy and bearing the granular cells showing the highest density. Estrus GFAP positive astrocytes in the stage of a high concentration of cells with specific cell body and appendages branched, Metestrus and Diestrus stage astrocytes are small and have short branches and are the lowest level of GFAP staining (Table 1).

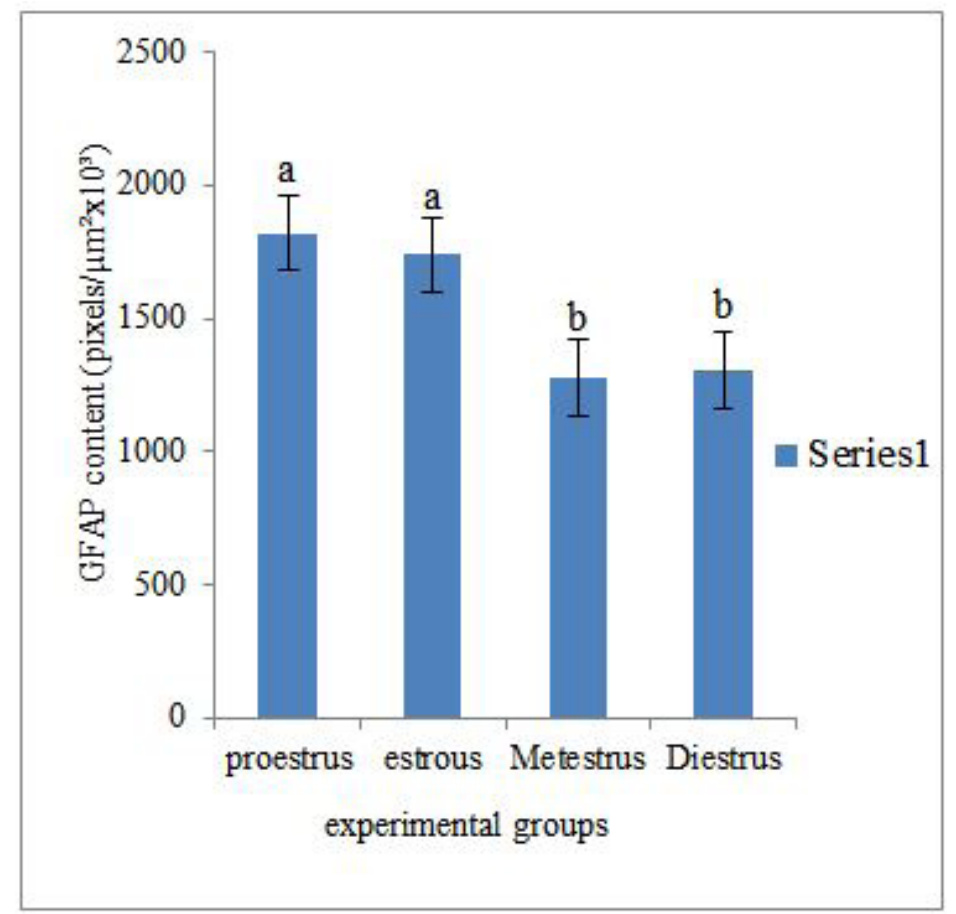

Table 1: Marker GFAP content in the hippocampus of mice at different stages of the Estrous cycle. In terms of content marker GFAP two Proestrus and Estrus phase with no significant differences $(\mathrm{p}<0.05)$, but with two stages Metestrus and Diestrus are significant. Pro: Proestrus stage, Es: Estrus stage, Met: Matestrus stage, Di: Diestrus stage

\section{The results measured sex hormones}

Estrogen: estrogen concentrations in the Proestrus stage of the maximum and minimum is in the stage of Diestrus. Estrogen concentrations in the Proestrus stage with other stages of the Estrous cycle are a significant difference. Estrogen levels during estrous, Metestrus and Diestrus has no significant difference $(\mathrm{p}<0.05)$ (Table 2$)$. 


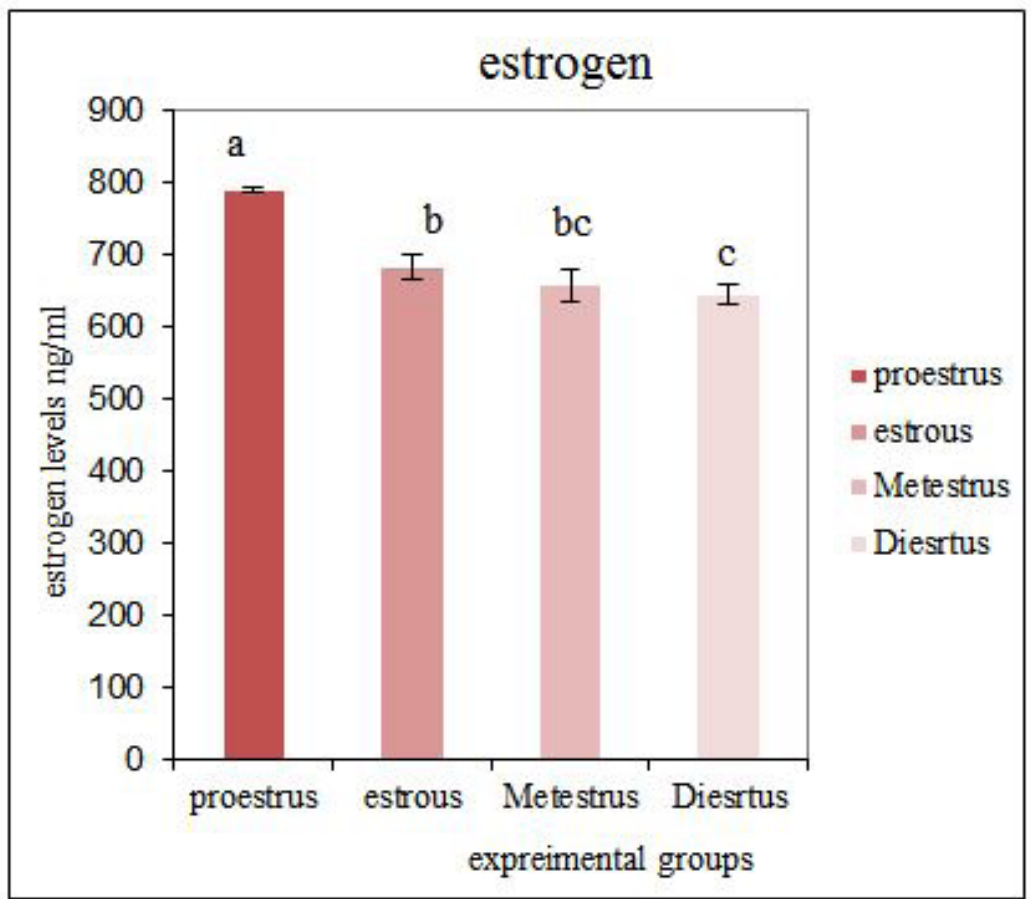

Table 2: Measurement of estrogen at different stages of the Estrous cycle .Estrous cycle stages with a significant difference between groups, groups and c, b and bc are significant differences together. Statistically, $\mathrm{p}<0.05$ was considered as significant level

Progesterone: Measuring progesterone highest and lowest concentrations of progesterone on Diestrus phase of the Proestrus phase. Progesterone in four stages of Estrous cycle has different dynamics and four groups a, b, and $c$ and $d$ are significant differences $(\mathrm{p}<0.05)$ (Table 3).

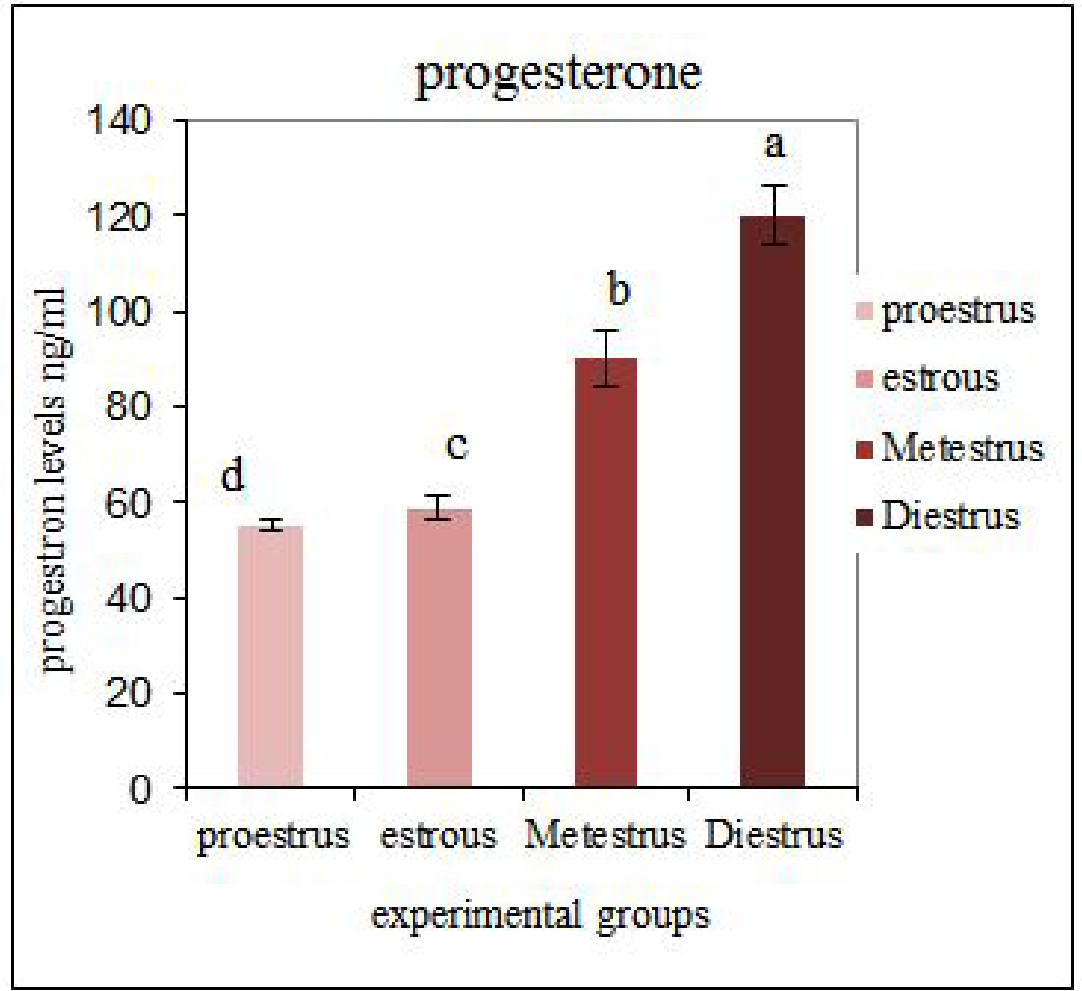

Table 3: Measurement of progesterone in different stages of the Estrous cycle .progesterone levels in four groups $\mathrm{a}, \mathrm{b}$, and $\mathrm{c}$ and $\mathrm{d}$ are significantly different. Proestrus stage and the stage of Diestrus its concentration is the lowest concentration

FSH: hormone FSH is similar to the hormone estrogen in the Proestrus maximum concentration of FSH in the Diestrus is minimal, the concentration of FSH in two phases Metestrus and Diestrus meaningful difference $(\mathrm{p}<0.05)($ Table 4$)$. 


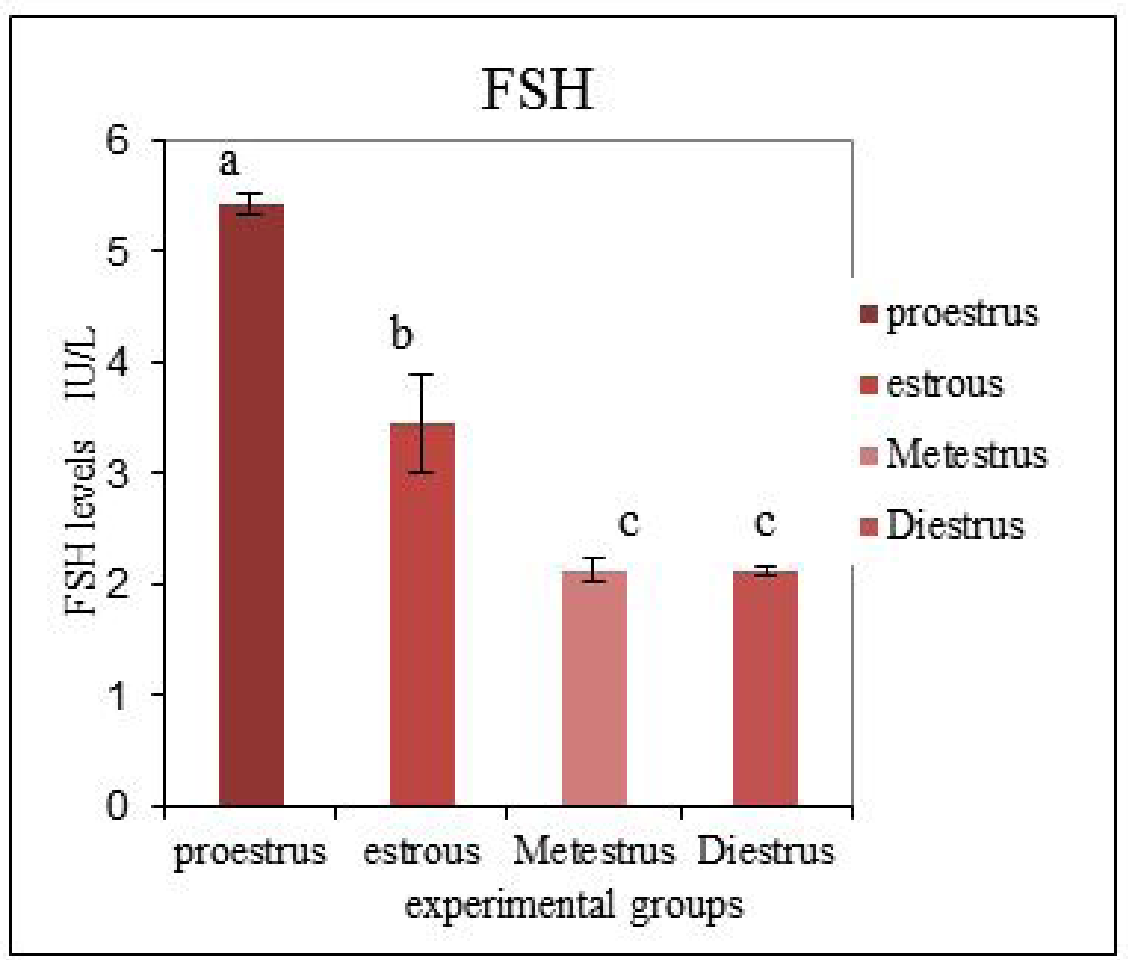

Table 4: Measurement of FSH in the various stages of the estrous cycle. The maximum concentration of a stage is Proestrus. Concentration in two phases $c$ and $d$ Metestrus significant difference $p<0.05$, a significant difference with the other stages of the Estrous cycle, Values international units per liter (IU / L). Statistically, p $<0.05$ was considered as significant level

LH: hormone LH likes the hormone estrogen and FSH in the Proestrus maximum. In Estrous phase concentrations of LH is minimal, Hormone LH in Metestrus and Diestrus two no significant difference $(\mathrm{p}<0.05)$ (Table 5).

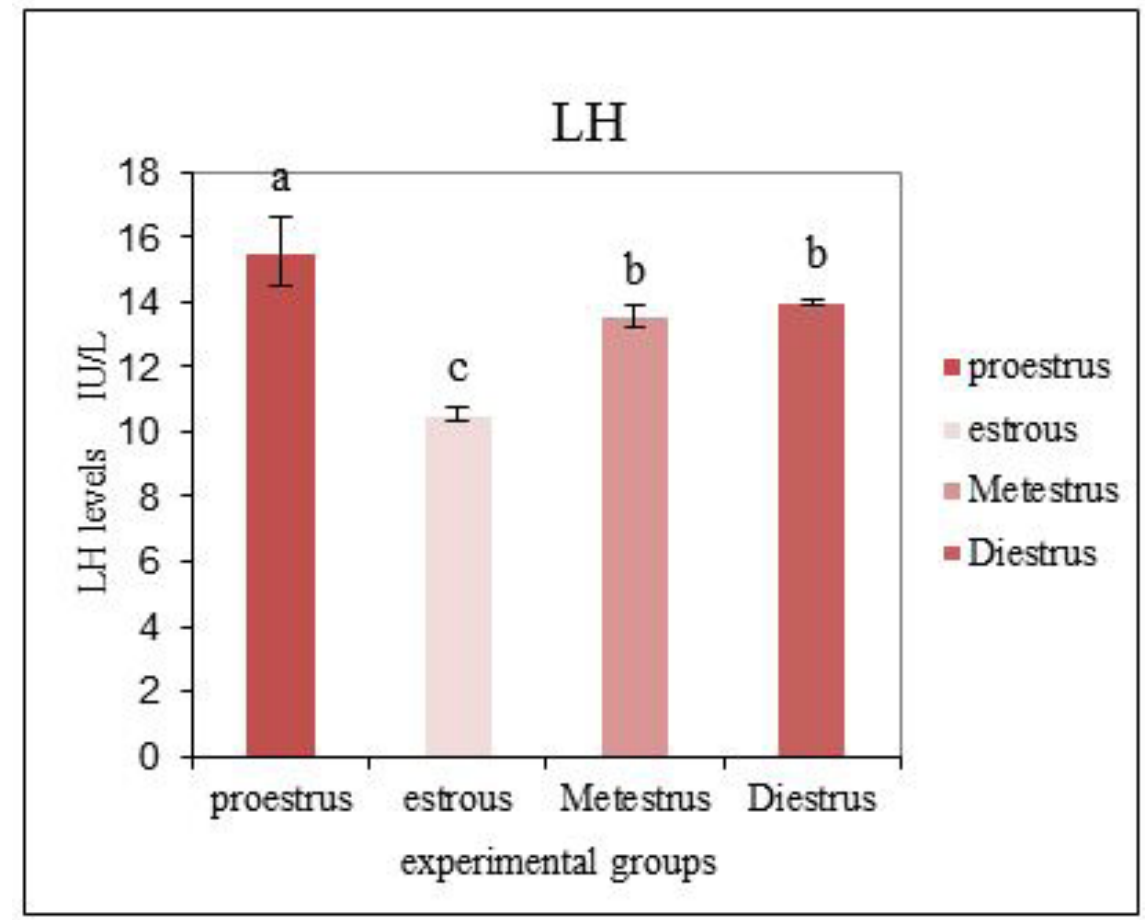

Table 5: LH hormone assays in various stages of the Estrous cycle. Maximum concentrations of LH on a stage of Proestrus and estrus c is minimal, LH concentration in step b Metestrus and Diestrus no significant difference

Prolactin: hormone prolactin concentration is Maximum in Estrus phase. The concentration of prolactin in Two phases Metestrus Diestrus showed no significant difference $(\mathrm{p}<0.05)$ (Table 6). 


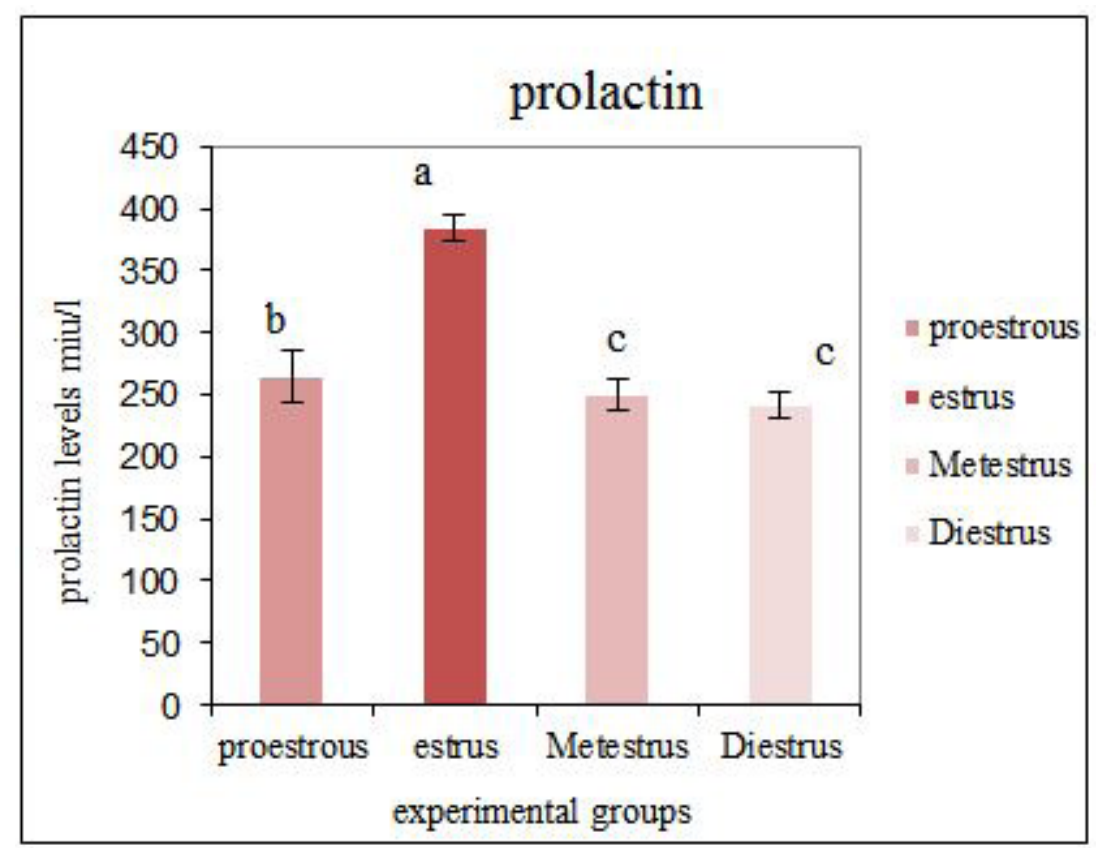

Table 6: Measurement of prolactin in various stages of the Estrous cycle. The maximum concentration of the hormone prolactin is a step Estrus, Metestrus prolactin concentration in stage $\mathrm{c}$ and d. Estrous showed no significant difference

\section{Discussion}

In this study Hippocampal Immunohistochemistry for GFAP markers were evaluated. The highest number of cells and staining in the Proestrus stage and were among the lowest in Estrous and Diestrus, Metestrus. One of the reasons for the increase markers of astrocytes in response to high concentrations of estrogen at this is Proestrus stage (Figure 2). The hormone progesterone in Proestrus stage of all stages is less (Figure3). Proestrus stage in the vaginal smear with nucleated epithelial cells dominate is determined, this stage of the day before ovulation when estrogen plasma at the highest level (Figure2) Associated. Estrus-stage squamous cell stratum is determined. Metestrus stage that combines three types of cells: leukocytes and nucleated epithelial cells or cornified epithelial cells. At this stage the serum estrogen level is low (Figure2). Diestrus stage, leukocytes are the predominant cells [14]. But in Proestrus and estrus, estrogen levels are high (Figure2). Proestrus period between analyzes of corpus luteum in the previous cycle and the new cycle and during the Proestrus stage follicular growth graph is completed, the vaginal smear often composed of nucleated epithelial cells $[15,16]$. The results showed that the number of cells with the marker GFAP positive reaction to the change of estrous cycle and fluctuating hormones especially estrogen change. Estrogen in Proestrus stage, followed by the highest levels of GFAP-positive cells in the SGZ region had the largest number among the estrous cycle. This could be a sign of increased neurogenesis in Proestrus compared to other stages, GFAP positive density in the hippocampus of mice during estrous cycle oscillation. In Proestrus phase, when estrogen levels go up, GFAP-positive cells also increased [17]. Hippocampal neurogenesis in mice throughout life in response to fluctuations steroid hormones during the estrous cycle, reproduction, stress and aging occurs. Suppress neurogenesis in areas that have been impaired as a result of nerve damage is important. Many of the effects of physiological and behavioral estrogen Mediated ovarian hormone progesterone may apply. Fluctuations in estrogen levels during the Estrous cycle and increasing the concentration of estrogen-induced LH concentrations are associated with the regulatory role of progesterone. The specific role of progesterone in the regulation of hippocampal neurons is not entirely clears, but signs of hippocampal neurogenesis by the hormone estrogen on their performance [18]. There are many reasons that Progesterone also has an effect on hippocampal neurons, for example, Progesterone receptor mRNA in the hippocampal dentate gyrus region and granular sub-region is located [19].

Progesterone inhibits protein synthesis and non-acceptance of sex as well. In general, estrogen and progesterone as protective antioxidant mechanisms of neuronal damage increase and decrease inflammation by altering glutamate receptor activity and increased nervous system synapses between neurons and neurotrophic support are provided. McEwen showed that the density of dendritic spines in the hippocampal pyramidal cells of adult female rats in Proestrus stage, which is the maximum concentration of estrogen, is higher-density, performance improvements and synaptic transmission is observed. Prior to treatment with NMDA receptor antagonists that requires activation of estrogen receptor and that this protective effect is mediated [20].

The Results also Hippocampus response to sex steroid hormones, particularly estrogen and progesterone in the morphology and the density of GFAP marker is well evident. During Proestrus high estrogen and progesterone hormone levels in the lowest stage, cell proliferation in the SGZ compared to Metestrus and Diestrus, when estrogen levels are low and progesterone is high, increased [21]. In immunofluorescent image (4-A) Proestrus stage seen Rising response to this marker and its presence in the 
SGZ Witness effects of estrogen on the hippocampal structurem. The brain as an organ is Dimorphism purpose of sex steroids, Gennady steroids to affect neurogenesis and it seems that cell proliferation in the presence of high concentrations of estrogens and progesterone concentration rises slightly [22]. SGZ area part of the hippocampus and one of the two main areas of the hippocampus neurogenesis in the adult brain, histologically a narrow strip of cells make up that Layer between granules (granular) and Hilus the dentate gyrus. Progenitor cells in the area are divided and daughter cells, mature granule cells make up [23]. Observations show that natural fluctuations of sex hormones in adult neurogenesis in several brain regions affected [24]. Neurogenesis in the hippocampus of adult male and female rats with altered levels of steroid hormones in circulation [25].

During the estrous cycle in the female rat hippocampal brain. In addition to differences in the morphology of astrocytes and density differences are dependent on the estrous cycle, concentration of estrogen, progesterone and expression of GFAP is also subject to change. Change the density surface this allows the astrocytes that synaptic plasticity in neurons is involved. Immunohistochemical studies by researchers in the front area of the hypothalamus the core POA showed, changes in the synapses between neurons along with changes in the density and number of astrocytes during the estrous cycle. Astrocytes can affect the secretion of hormones $\mathrm{GnRH}$ from the hypothalamus and the increase in LH. The surface density of astrocytes before the release of GnRH and LH sudden increase in Proestrus is vast and the number of astrocytes in this stage than other stages, with the passage of time in estrous phase reduced surface density and number of astrocytes [26]. Important role in maintaining homeostasis of the nervous tissue astrocytes play, Astrocytes express estrogen receptors and in activities neuroprotective brain are involved. In addition Neuroprotective activity of estrogen, astrocytes are also the source of the nerve agent. Estrogen via the aromatase enzyme in the brain can be built locally. Season sex in some mammal's volatility the steroid hormones causes neurological changes and neurogenesis to adapt to the environment. In times of stress, for survival partial response in the brain and body to limit the metabolic caused [27,28].

\section{Conclusion}

Morphometric study marker GFAP expression in the dentate gyrus of the hippocampus And measurement of steroid hormones during the estrous cycle showed that in Proestrus stage, the concentration of sex hormones such as estrogen, FSH and LH is high. High concentrations of these hormones in conjunction with concentrated expression of GFAP in the area SGZ in Proestrus stage, indicating a direct relationship between the sex hormones estrogen on increasing neurogenesis is that it plays an important role. Fluctuations in estrogen and its permanent increase in neurogenesis is more of a high concentration of the mediating role of progesterone in causing this volatility is very important. Unlike other steroid hormones progesterone concentration is lowest in Proestrus stage; this small concentration of progesterone mediated by estrogen affects performance and increases the effect of estrogen on the expression of GFAP in the area SGZ in the Proestrus stage.

\section{Acknowledgements}

Author thankful Damghan University of biology laboratory director Mr. Kosha and Ms. Azeri

\section{Funding}

This study was supported by a grant from School of Biology and Institute of Biological Sciences of Damghan University.

\section{Ethics approval and consent to participate}

This study was approved by the Animal Research Ethics Board, University of Damghan (Animal Use Protocol \# 20130068). There was no human participation in this study.

\section{Conflict of Interests}

The author declares that they have no competing interests.

\section{Authors' information}

In the Figure 7 the microscopic sections of the hippocampus of mice at different stages of the estrous cycle phase contrast image on the left is the right immunofluorescent images.

\section{Reference}

1. Spencer JL, Waters EM, Milner TA, McEwen BS (2008) Estrous cycle regulates activation of hippocampal Akt, LIM kinase, and neurotrophic receptor in C57BL/6 mice. Neuroscience 155: 1106-19.

2. Barron ML, Flick LH, Cook CA, Homan SM, Campbell C (2008) Associations between Psychiatric Disorders and Menstrual Cycle Characteristics. Arch Psychiatr Nurs 22: 254-65.

3. Villa A, Vegeto E, Poletti A, Maggi A (2016) Estrogens, Neuroinflammation and Neurodegeneration. Endocr Rev 37: $372-402$.

4. Galea LA, Spritzer MD, Barker JM, Pawluski JL (2006) Gonadal Hormone Modulation of Hippocampal Neurogenesis in the Adult. Hippocampus 16: 225-32.

5. Spencer JL, Waters EM, Romeo RD, Wood GE, Milner TA, et al. (2008) Uncovering the mechanisms of estrogen effects on hippocampal function. Front neuroendocrinol 29: 219-37. 
6. Cora MC, Kooistra L, Travlos G (2015) Vaginal cytology of the laboratory rat and mouse: review and criteria for the staging of the estrous cycle using stained vaginal smears. Toxicol Pathol 43: 776-93.

7. Westwood FR (2008) The female rat reproductive cycle: A practical histological guide to staging. Toxicol Pathol 36: $375-84$.

8. Fowler CD, Liu Y, wang Z (2008) Estrogen and adult neurogenesis in the amygdala and hypothalamus. Brain Res Rev 57: $342-51$.

9. Kempermann G, Song H, Gage FH (2015) neurogenesis in the adult hippocampus. Cold Spring Harb Perspect Biol. 7: a018812.

10. McEwen BS (1985) Steroids and brain Function. Trends Pharmacol Sci 6: 22-6.

11. Mak GK, Enwere EK, Gregg C, Pakarainen T, Poutanen M, et al. (2007) Male pheromone-stimulated neurogenesis in the adult female brain: possible role in mating behavior. Nat Neurosci 10: 1003-11.

12. Peper JS, Brouwer RM, Schnack HG, van Baal GC, van Leeuwen M, et al. (2009) Sex steroids and brain structure in pubertal boys and girls. Psychoneuroendocrinology 34: 332-42.

13. Pawluski JL, Brummelte S, Barha CK, Crozier TM, Galea LA (2009) Effects of steroid hormones on neurogenesis in the hippocampus of the adult female rodent during the estrous cycle, pregnancy, lactation and aging. Front Neuroendocrinol 30: 343-57.

14. Mazzucco CA, Lieblich SE, Bingham BI, Williamson MA, Viau V, et al. (2006) Both estrogen receptor alpha and estrogen receptor beta agonists enhance cell proliferation in the dentate gyrus of adult female rats. Neuroscience 141: 1793-800.

15. Barha CK, Galea LA (2010) fluence of different estrogens on neuroplasticity and cognition in the hippocampus. Biochim Biophys Acta $1800: 1056-67$.

16. Champlain AK, Dorr DL, Gates AH (1973) Determining the stage of the estrous cycle in the mouse by the appearance of the vagina. Boil Reprod 8: 491-4.

17. Arias C, Zepeda A, Hernández-Ortega K, Leal-Galicia P, Lojero C, et al. (2009) Sex and estrous cycle-dependent differences in glial fibrillary acidic protein immunoreactivity in the adult rat hippocampus. Horm Behav, 2009. 55: 257-63.

18. Kuhn HG, Dickinson-Anson H, Gage FH (1996) Neurogenesis in the Dentate Gyrus of the Adult Rat: Age-Related Decrease of Neuronal Progenitor Proliferatio. J Neurosci 16: 2027-33.

19. Hagihara K, Hirata S, Osada T, Hirai M, Kato J (1992) Distribution of cells containing progesterone receptor mRNA in the female rat di-and telencephalon. Brain Res Mol Brain Res. 14: 239-49.

20. Stein DG (2001) Brain damage, sex hormones and recovery: a new role for progesterone and estrogen? Trends in Neurosciences 24 : $386-90$.

21. Lennington JB, Yang Z, Conover JC (2003) Neural stem cells and the regulation of adult neurogenesis. Reprod Biol Endocrinol 1: 99.

22. Heberden C (2017) Sex steroids and neurogenesis. Biochem Pharmacol 141: 56-62.

23. Ming GL, Song H (2005) Adult neurogenesis in the mammalian central nervous system. Annu Rev Neurosci 28: 223-50.

24. Tanapat P, Hastings NB, Reeves AJ, Gould E (1999) Estrogen stimulates a transient increase in the number of new neurons in the dentate gyrus of the adult female rat. J Neurosci 19: 5792-801.

25. Leuner B, Sabihi S (2016) the birth of new neurons in the maternal brain: Hormonal regulation and functional implications. Front Neuroendocrinol 41 : $99-113$.

26. Cashion AB, Smith MJ, Wise PM (2003) The Morphometry of Astrocytes in the Rostral Preoptic Area Exhibits a Diurnal Rhythm on Proestrus: Relationship to the Luteinizing Hormone Surge and Effects of Age.Endocrinology 144: 274-80.

27. Brown AM, Ransom BR (2007) Astrocyte glycogen and brain energy metabolism. Glia 55: 1263-71.

28. Duarte-Guterman P, Yagi S, Chow C, Galea LA (2015) Hippocampal learning, memory, and neurogenesis: Effects of sex and estrogens across the lifespan in adults. Horm Behav 74: 37-52.

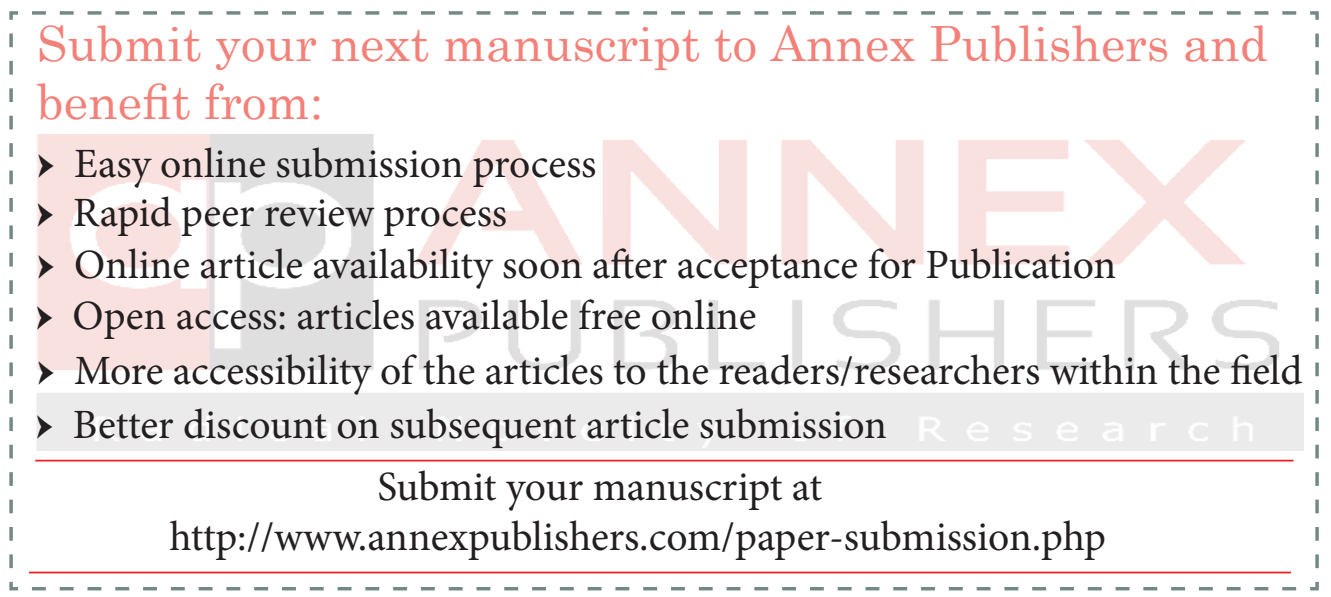

